J. Austral. Math. Soc. 22 (Series A) (1976), 282-304.

\title{
OSCILLATORY AND ASYMPTOTIC PROPERTIES OF HOMOGENEOUS AND NONHOMOGENEOUS DELAY DIFFERENTIAL EQUATIONS OF EVEN ORDER
}

\author{
RAYMOND D. TERRY
}

(Received 8 April 1975)

\begin{abstract}
In this paper we consider the (non)oscillation properties of two general nonhomogeneous nonlinear delay differential equations of order $2 n$

$$
D^{n}\left[r(t) D^{n} y(t)\right] \pm y_{r}(t) f\left[t, y_{r}(t)\right]=O(t),
$$

using as background and motivation the techniques previously applied to the associated homogeneous delay differential equations $H_{+}$and $H_{-}$. The equations $N_{+}$and $N_{-}$are each reduced to homogeneous form by the introduction of transformations $u(t)=y(t)-R(t)$ and $v(t)=R(t)-y(t)$, where $R(t)$ is a solution of the associated nonhomogeneous differential equation $(N)$. We first extend certain results for the equation $H_{+}$and then develop a classification of the positive solutions of equation $H_{-}$. Using this classification and the one developed by Terry (1974) for $H_{\text {, we develop a }}$ natural classification of the positive solutions of $N_{+}$and $N_{-}$according to the sign properties of the derivatives of $u(t)$ and $v(t)$. For each choice of $R(t)$, it is seen that there are $2 n+1$ types of positive solutions of $N_{+}$or $N_{-}$. An intermediate Riccati transformation is employed to obtain integral criteria for the nonexistence of some of these solutions. Analysis of the Taylor remainder results in sufficient conditions for the nonexistence of other such solutions.
\end{abstract}

The purpose of this paper is to discuss the oscillatory and nonoscillatory behavior of solutions of the nonlinear delay differential equations of order $2 n$ :

$$
D^{n}\left[r(t) D^{n} y(t)\right]+y_{\tau}(t) f\left[t, y_{\tau}(t)\right]=Q(t)
$$

and

$$
D^{n}\left[r(t) D^{n} y(t)\right]-y_{\tau}(t) f\left[t, y_{\tau}(t)\right]=Q(t),
$$

where $Q(t) \not \equiv 0,0<m \leqq r(t) \leqq M, y_{\tau}(t)=y[t-\tau(t)]$ and $0 \leqq \tau(t)<t$. Throughout the paper $f(t, u)$ is assumed to satisfy the following three hypotheses:

(i) $f(t, u)$ is a continuous real valued function on $[0, \infty) \times R, R=(-\infty, \infty)$;

(ii) for each fixed $t \in[0, \infty), f(t, u)<f(t, v)$ for $0<u<v$; and 
(iii) for each fixed $t \in[0, \infty), f(t, u)>0$ and $f(t, u)=f(t,-u)$ for $u \neq 0$. Before considering the nonhomogeneous equations $N_{+}$and $N_{-}$we first review and extend some results concerning the associated equations

$$
\begin{gathered}
D^{n}\left[r(t) D^{n} y(t)\right]+y_{\tau}(t) f\left[t, y_{\tau}(t)\right]=0 \text { and } \\
D^{n}\left[r(t) D^{n} y(t)\right]-y_{\tau}(t) f\left[t, y_{\tau}(t)\right]=0 .
\end{gathered}
$$

A solution $y(t)$ of $H_{+}, H_{-}, N_{+}$or $N_{-}$is said to be oscillatory on $[a, \infty)$ if for each $\alpha>a$, there exists a $\beta>\alpha$ such that $y(\beta)=0$; it is called nonoscillatory otherwise. Following Terry (1974), we say that a solution of $H_{+}$is of type $B_{j}$ if for sufficiently large $t, y_{k}(t)>0$ for $k=0, \cdots, 2 j+1$ and $(-1)^{k+1} y_{k}(t)>0, k=$ $2 j+2, \cdots, 2 n-1$ where

$$
y_{k}(t)=\left\{\begin{array}{cl}
D^{k} y(t), & k=0, \cdots, n-1 \text { and } \\
D^{k-n}\left[r(t) D^{n} y(t)\right], & k=n, \cdots, 2 n-1 .
\end{array}\right.
$$

For definiteness, we say that $y(t)$ is of type $B_{j}$ on $\left[T_{0}, \infty\right)$ if the $y_{k}(t)$ have the appropriate sign properties for $t \geqq T_{0}$. It has been shown in Terry (1974) that a positive solution of $H_{+}$is necessarily of type $B_{i}$ for some $j=0, \cdots, n-1$. Moreover, under the assumption that $0 \leqq \tau(t) \leqq T<\infty$, the following lemmas were proved:

Lemma 1.1. Let $y(t)$ be a $B_{i}$-solution of $H_{+}$on $\left[T_{0}, \infty\right)$. Then there exist positive constants $N_{k, k-1}$ such that

$$
\left(t-T_{1}\right) y_{k}(t) \leqq N_{k, k-1} y_{k-1}(t), k=1, \cdots, 2 j+1
$$

for $t \geqq T_{1}=T_{0}+T$.

Lemma 1.2. Let $y(t)$ be a $B_{j}$-solution of $H_{+}$. Then there exist constants $K_{i}>0$ and $t_{i}>0$ such that

$$
\frac{y_{i \tau}(t)}{y_{i}(t)} \geqq K_{i}, i=0, \cdots, 2 j
$$

for $t \geqq t_{i}$.

In an analogous manner we may define a solution of $H_{-}$to be of type $\mathscr{B}_{j}(0 \leqq j \leqq n-1)$ if for $t$ sufficiently large, $y_{k}(t)>0, \quad k=0, \cdots, 2 j$ and $(-1)^{k} y_{k}(t)>0, k=2 j+1, \cdots, 2 n-1$. A solution is of type $\mathscr{B}_{n}$ if $y_{k}(t)>$ $0, \cdots, 2 n-1$ for large $t$. We observe that when $n=2, \tau(t) \equiv 0$ and $r(t) \equiv 1$, the solutions of type $\mathscr{B}_{0}$ reduce to those investigated in Wong (1969), where they 
were referred to as proper solutions of type I. The solutions of type $\mathscr{B}_{1}$ and $\mathscr{B}_{2}$ were collectively referred to as proper solutions of type II. It is easily seen that a positive solution of $H_{-}$is necessarily of type $\mathscr{B}_{j}$ for some $j=0, \cdots, n$ and that the following analogues of Lemmas 1.1 and 1.2 can be derived when $1 \leqq j \leqq$ $n-1$.

Lemma 1.1.' Let $y(t)$ be a $\mathscr{B}_{i}$-solution of $H_{-}$on $\left[T_{0}, \infty\right)$. Then there exist positive constants $\eta_{k, k-1}$ such that

$$
\left(t-T_{1}\right) y_{k}(t) \leqq \eta_{k, k-1} y_{k-1}(t), \quad k=1, \cdots, 2 j
$$

for $t \geqq T_{1}=T_{0}+T$.

Lemma 1.2.' Let $y(t)$ be a $\mathscr{B}_{j}$-solution of $H_{-}$. Then there exists constants $\kappa_{i}>0$ and $t_{i}>0$ such that

$$
\frac{y_{i \tau}(t)}{y_{i}(t)} \geqq \kappa_{i}, \quad i=0, \cdots, 2 j-1
$$

for $t \geqq t_{i}$.

The proof of Lemma 1.1' depends only on the technique of integration by parts and the definition of a $\mathscr{B}_{j}$-solution and is an imitation of the proof of Lemma 1.1. Moreover, the proof of Lemma 1.2' follows from Lemma 1.1' in the same manner that Lemma 1.2 followed from Lemma 1.1 in Terry (1974).

In Section two the basic lemmas given here are extended to the case where $\tau(t)$ is unbounded in a prescribed manner. The resulting lemmas are then used to obtain integral criteria for the nonexistence of solutions of $H_{+}$of type $B_{j}$ as well as for the oscillation of all solutions of $H_{+}$. Section three provides sufficient

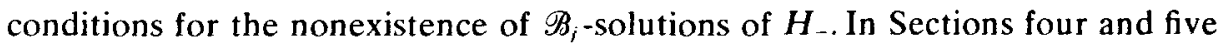
we let $R(t)$ be a solution of the nonhomogeneous differential equation

$$
D^{n}\left[r(t) D^{n} y(t)\right]=Q(t)
$$

for each choice of $R(t)$, the positive solutions of $N_{+}$or $N_{-}$may be classified according to $2 n+1$ types. The methods of the previous sections are then employed to exclude some of these solutions. If, for a specific choice of $R(t)$, conditions can be given to exclude all $2 n+1$ types, we can conclude that $N_{+}$(or $N_{-}$) has no positive solutions. We note that even when this is not possible the exclusion of some of the solutions will necessarily give information concerning the asymptotic behavior of the remaining types. With each choice of $R(t)$ additional information is obtained concerning the behavior of the functions $y_{k}(t)$ for large $t$. The results are valid for ordinary differential equations as well and are applied to show the nonexistence of bounded positive solutions of certain equations or to show that bounded positive solutions tend to zero ultimately. 
The homogeneous equation $H_{+}$has been studied by Terry and Wong (1972) when $n=2$ and by Terry (1974) for arbitrary $n$. A slightly different form of $H_{+}$ has been considered by Ladas (1971b) when $r(t) \equiv 1$. An initial investigation of the oscillation and separation properties of the nonhomogeneous equation is due to Burton (1952) for the case $\tau(t) \equiv 0, n=1$ and $f(t, u)=p(t) u(t)$. More recent studies include those by Howard (to appear) for the second order differential equation and by Kartsatos (1971) and Kartsatos (1972) in the $n$-th order case when the forcing term is assumed to be small or periodic.

Since submitting the first draft of this paper, additional papers on nonhomogeneous delay differential equations have been authored by Kusano (1973), Kusano and Onose (1974), Singh (to appear), Singh and Dahiya (to appear) and others.

In this section we extend the basic lemmas of section one to the case where $\lim _{t \rightarrow \infty} \tau(t)=\lim _{t \rightarrow \infty}[t-\tau(t)]=\infty$. Specifically, $\tau(t)$ is assumed to satisfy either

$$
\begin{gathered}
0 \leqq \tau(t) \leqq \mu t, 0 \leqq \mu<1 \text { or } \\
0 \leqq \tau(t) \leqq \mu t^{\beta}, 0 \leqq \mu<\infty \text { and } 0 \leqq \beta<1 .
\end{gathered}
$$

Lemma. 2.1. Suppose $\tau(t)$ satisfies (T1) or (T2) and that $y(t)$ is a $B_{i}$-solution of $H_{+}$on $\left[T_{0}, \infty\right)$. Then there exist constants $N_{k, k-1}>0$ such that $\left(t-T_{1}\right) y_{k}(t) \leqq N_{k, k-1} y_{k-1}(t)$ for $t \geqq T_{1}$ and $t y_{k}(t) \leqq 2 N_{k, k-1} y_{k-1}(t)$ for $t \leqq 2 T_{1}$, where

$$
T_{1} \geqq \min \left\{t \geqq T_{0}: t-\tau(t) \geqq T_{0} \text { for } t \geqq T_{1}\right\}
$$

Proof. Suppose that $y(t)$ is a solution of $H_{+}$of type $B_{j}$ on [ $\left.T_{o}, \infty\right)$. If $\tau(t)$ satisfies (T1), then $t-\tau(t) \geqq(1-\mu) t$. On the other hand, if $\tau(t)$ satisfies (T2), then

$$
t-\tau(t) \geqq t-\mu t^{\beta}=t^{\beta}\left[t^{1-\beta}-\mu\right] .
$$

Let $\varepsilon$ be chosen so that $0<\varepsilon<1$. Since

$$
\lim _{t \rightarrow \infty} \frac{t^{1-\beta}-\mu}{t^{1-\beta}}=1
$$

$t^{1-\beta}-\mu \geqq(1-\varepsilon) t^{1-\beta}$ for $t$ sufficiently large. Hence, there is a $T_{*} \geqq T_{0}$ such that

$$
t-\tau(t) \geqq t^{\beta}\left[(1-\varepsilon) t^{1-\beta}\right]=(1-\varepsilon) t
$$


for $t \geqq T_{*}$. It follows that there is a $T_{1} \geqq T_{*}$ such that $t-\tau(t) \geqq T_{0}$ for $t \geqq T_{1}$. For the assumption (T2), $T_{1}=\max \left(T_{*},(1-\varepsilon)^{-1} T_{0}\right)$. For (T1), $T_{1}=(1-\mu)^{-1} T_{0}$. The proof given in Terry (1974) is now valid verbatim with the estimates holding for $t \geqq T_{1}$. As before, we obtain the intermediate inequalities

$$
\left(t-T_{1}\right) y_{k}(t) \leqq N_{k, k-1} y_{k-1}(t), k=1, \cdots, 2 j+1
$$

for $t \geqq T_{1}$.

We note that the estimates on the constants $N_{k, k-1}$ are the same as given in Terry (1974) since the estimates are obtained as part of the proof.

LEMMA 2.2. Let $y(t)$ be a $B_{j}$-solution of $H_{+}$. Let $i$ be an integer $(0 \leqq i \leqq 2 j)$ and $\tau(t)$ satisfy (T2) or (T1), where $\mu<\left[m^{-1} N_{n, n-1}+1\right]^{-1}$, if $n$ is odd, $j \geqq$ $(n-1) / 2$ and $\left.i=n-1 ; \mu<\left[N_{i+1, i}+1\right)^{-1}\right)^{-1}$, otherwise. Then there exist positive constants $K_{i}$ and $t_{i}$ such that

$$
y_{i_{r}}(t) \geqq K_{i} y_{i}(t), t \geqq t_{i} \quad(i=0, \cdots, 2 j) .
$$

Proof. Let $y(t)$ be a $B_{j}$-solution of $H_{+}$on $\left[T_{0}, \infty\right)$. Suppose $i \neq n-1$ if $n$ is odd. Then $y(t), y_{\tau}(t), y_{k}(t)(k=1, \cdots, 2 j+1)$ are all positive for $t \geqq T_{1}$ (chosen as in Lemma 2.1). Since $\tau(t) \geqq 0, y_{i_{\tau}}(t) \equiv y_{i}[t-\tau(t)] \leqq y_{i}(t)$ for $i=0, \cdots, 2 j$. Moreover, $s-T_{1} \geqq t-\tau(t)-T_{1}$ for any $s$ in the interval $J_{\tau}(t)=[t-\tau(t), t]$. An application of Lemma 2.1 and a mean-value theorem shows that there is an $s \in J_{\tau}(t)$ such that

$$
\begin{aligned}
\left|\frac{y_{i \tau}(t)}{y_{i}(t)}-1\right| & =\frac{\left|y_{i \tau}(t)-y_{i}(t)\right|}{\left|y_{i}(t)\right|}=\frac{y_{i}(t)-y_{i \tau}(t)}{y_{i}(t)} \\
& =\tau(t) \frac{y_{i}^{\prime}(s)}{y_{i}(t)} \leqq \frac{\tau(t)}{s-T_{1}} N_{i+1, i} \frac{y_{i}(s)}{y_{i}(t)} \\
& \leqq N_{i+1, i} \frac{\tau(t)}{t-\tau(t)-T_{1}} .
\end{aligned}
$$

We first consider the condition (T1). Suppose that $0 \leqq \tau(t) \leqq \mu t, 0 \leqq \mu<$ $\left[N_{i+1, i}+1\right]^{-1}$. Then we define $\delta$ by

$$
\mu=\left[N_{i+1, i}+1\right]^{-1}-\delta,
$$

where $0<\delta<\left[N_{i+1, i}+1\right]^{-1}<1$. Let $\alpha>1$ and define $\varepsilon>0$ by

$$
\delta=\frac{\alpha \varepsilon N_{i+1,1}}{\left(N_{i+1, i}+1\right)\left(N_{i+1, i}+1-\alpha \varepsilon\right)} .
$$

It follows that

$$
0<\alpha \varepsilon=\frac{\left(N_{i+1, i}+1\right)^{2} \delta}{N_{i+1, i}+\left(N_{i+1, i}+1\right) \delta}
$$


This expression is of the form $b^{2} \delta(a+b \delta)^{-1}$, where $a=N_{i+1, i}$ and $b=a+1$. Since $b \delta<1, a b \delta<a$. Also, since $b=a+1$,

$$
b^{2} \delta=b(a+1) \delta=b \delta+a b \delta<b \delta+a
$$

and $\alpha \varepsilon=b^{2} \delta(a+b \delta)^{-1}<1$. Since $\alpha>1, \varepsilon<\alpha^{-1}<1$.

For this choice of $\varepsilon$ and for $t$ sufficiently large $\left(t \geqq t_{i}\right)$

$$
\begin{aligned}
N_{i+1, i} \frac{\tau(t)}{t-\tau(t)-T_{1}} & \leqq N_{i+1, i} \frac{\mu t}{(1-\mu) t-T_{1}}=\frac{N_{i+1, i} \mu t}{(1-\mu)\left[t-T_{1} /(1-\mu)\right]} \\
& \leqq N_{i+1, i} \frac{1}{1-\varepsilon} \frac{\mu}{1-\mu} .
\end{aligned}
$$

Then

$$
\mu=\frac{1}{N_{i+1, i}+1}-\delta=\frac{1-\alpha \varepsilon}{N_{i+1, i}+(1-\alpha \varepsilon)} \text { and } \frac{y_{i}(s)}{s-T_{1}}=\frac{1-\alpha \varepsilon}{N_{i+1, i}} .
$$

It follows that

$$
N_{i+1, i} \frac{\tau(t)}{t-\tau(t)-T_{1}} \leqq \frac{1-\alpha \varepsilon}{1-\varepsilon}<1, \quad t \geqq t_{i}
$$

and we may take $K_{i}=1-(1-\alpha \varepsilon) /(1-\varepsilon)$.

If $n$ is odd, $j \geqq(n-1) / 2$ and $i=n-1$,

$$
y_{i}^{\prime}(s)=D y_{n-1}(s)=\frac{y_{n}(s)}{r(s)}<\frac{y_{n}(s)}{m}<\frac{y_{i}(s)}{s-T_{1}} \frac{N_{n, n-1}}{m}
$$

we may repeat the procedure above replacing $N_{i+1, i}$ by $m^{-1} N_{n, n-1}$ and thus obtain the proof of the second assertion.

Now suppose $\tau(t)$ satisfies (T2). Let $\varepsilon_{1}$ and $\varepsilon_{2}$ be chosen so that $0<\varepsilon_{i}<1$, $i=1,2$. Since $\lim _{t \rightarrow \infty}[t-\tau(t)]=\infty$, we have $t-\tau(t)-T_{1} \geqq\left(1-\varepsilon_{1}\right)[t-\tau(t)]$ for $t$ sufficiently large. Since $0 \leqq \beta<1, \lim _{t \rightarrow \infty} t^{1-\beta}=\infty$ so that $t^{1-\beta}-\mu \geqq\left(1-\varepsilon_{2}\right) t^{1-\beta}$ for $t$ sufficiently large. Thus

$$
t-\tau(t) \geqq t-\mu t^{\beta}=t^{\beta}\left[t^{1-\beta}-\mu\right] \geqq\left(1-\varepsilon_{2}\right) t .
$$

Hence,

$$
\begin{aligned}
\frac{\tau(t)}{t-\tau(t)-T_{1}} & \leqq \frac{\tau(t)}{\left(1-\varepsilon_{1}\right)[t-\tau(t)]} \leqq \frac{\mu t^{\beta}}{\left(1-\varepsilon_{1}\right)\left(1-\varepsilon_{2}\right) t}=\frac{\mu}{\left(1-\varepsilon_{1}\right)\left(1-\varepsilon_{2}\right) t^{1-\beta}} \\
& <1-\varepsilon,
\end{aligned}
$$

if $t>\left[\mu(1-\varepsilon)^{-1}\left(1-\varepsilon_{t}\right)^{-1}\left(1-\varepsilon_{2}\right)^{-1}\right]^{1 /(1-\beta)}$. 
We now state an extended version of Theorem 2.5 of Terry (1974), which was previously proved for $\tau(t) \geqq 0$ and bounded.

THEOREM 2.3. Let $k$ be an integer $(k=0, \cdots, n-1)$. Let $\tau(t)$ satisfy (T2) or (T1) with

$$
\mu<\left[m^{-1} N_{n, n-1}+1\right]^{-1} \text {, if } n \text { is odd and } k=\frac{(n-1)}{2}
$$

or

$$
\mu<\left[N_{2 k+1,2 k}+1\right]^{-1} \text {, otherwise. }
$$

Suppose that for all constants $C>0$

$$
\int^{\infty} t^{2 k} f\left(t, C t^{2 k}\right) d t=+\infty .
$$

Then $H_{+}$has no solutions of type $B_{r}(r=k, \cdots, n-1)$.

The proof is accomplished by using the intermediate Riccati transformation $z(t)=u_{2 n-1}(t) u_{2 k}^{-1}(t)$ and is the same as in Terry (1974) except for the use of Lemmas 2.1 and 2.2 instead of Lemmas 1.1 and 1.2. The crucial step of the proof is the consideration of the term $u_{2 k, \tau}(t) u_{2 k}^{-1}(t)$. In the event that $\tau(t)$ satisfies (T1) with $0 \leqq \mu \leqq\left[N_{2 k+1,2 k}+1\right]^{-1}$, we may conclude by Lemma 2.2 that this term is bounded away from zero.

In attempting to eliminate $B_{j}$-solutions, where $j \geqq k+1$, we are led to consider the term $u_{2 j, r}(t) u_{2 j}^{-1}(t)$. We note, however, that if $y(t)$ is a $B_{k}$-solution of $H_{+}$and $k \neq(n-1) / 2$, then we may take $N_{2 k+1,2 k}=1$ : for in this case $y_{2 k+1}(t)$ is a positive decreasing function of $t$ for $t \geqq T_{1}$ and an integration from $T_{1}$ to $t$ shows that

$$
y_{2 k}(t)-y_{2 k}\left(T_{1}\right)=\int_{T_{1}}^{t} y_{2 k+1}(s) d s \geqq\left(t-T_{1}\right) y_{2 k+1}(t) .
$$

Since $y_{2 k}\left(T_{1}\right)>0,\left(t-T_{1}\right) y_{2 k+1}(t) \leqq y_{2 k}(t)$.

Similarly, if $y(t)$ is a $B_{i}$-solution of $H_{+}$, where $j \geqq k+1$, we may take $N_{2 j+1,2 j}=1$. If $k=(n-1) / 2$, then we may take $N_{2 k+1,2 k}=N_{n, n-1}=M$ since

$$
\begin{aligned}
\left(t-T_{1}\right) y_{n}(t) & =\left(t-T_{1}\right) y_{2 k+1}(t) \leqq \int_{T_{1}}^{t} y_{2 k+1}(s) d s=\int_{T_{1}}^{t} y_{n}(s) d s \\
& =\int_{T_{1}}^{t} r(s) D y_{n-1}(s) d s \leqq M \int_{T_{1}}^{t} D y_{n-1}(s) d s \\
& =M\left[y_{n-1}(t)-y_{n-1}\left(T_{1}\right)\right]<M y_{n-1}(t) .
\end{aligned}
$$


The condition $0 \leqq \mu<\left[m^{-1} N_{2 k+1,2 k}+1\right)^{-1}$ becomes $0 \leqq \mu<m /(m+M)$. If $j \geqq$ $k+1$, the required condition is $0 \leqq \mu<1 / 2$, which is already existent since $m /(m+M)<1 / 2$. Thus, we may replace the condition of Theorem 2.3 by the slightly stronger condition

$$
\begin{gathered}
0 \leqq \tau(t) \leqq \mu t, \text { where } \\
0 \leqq \mu<m(m+M)^{-1}, \text { if } n \text { is odd; } \\
0 \leqq \mu<1 / 2, \text { otherwise. }
\end{gathered}
$$

We will assume in the sequel that $\tau(t)$ satisfies either (T2) or (T3).

THEOREM 2.4. Let $\tau(t)$ satisfy (T2) or (T3) Suppose that for some $k=$ $0, \cdots, n-1$ and for all positive constants $C$

$$
\int^{\infty} t^{2 n-1} f\left(t, C t^{2 k}\right) d t=\infty .
$$

Then $H_{+}$has no positive $B_{k}$-solutions $y(t)$ such that $y_{2 k}(t)$ is bounded.

Proof. Suppose that $y(t)$ is a positive $B_{k}$-solution of $H_{+}$for $t \geqq T_{0}$. Then for $t \geqq T_{1}, y_{\tau}(t)$ and $y_{i}(t)$ are positive $(i=0, \cdots, 2 k+1)$ and $(-1)^{i+1} y_{i}(t)>$ $0(i=2 k+2, \cdots, 2 n-1)$. We note that the hypotheses on $\tau(t)$ are not used explicitly below. They are necessary only for the application of Lemma 2.1. Multiplying both sides of $H_{+}$by $t^{2 n-2 k-1}$ and integrating from $T_{1}$ to $t$ yields

$$
\int_{T_{1}}^{t} s^{2 n-2 k-1} D^{n}\left[r(s) D^{n} y(s)\right] d s+\int_{T_{1}}^{t} s^{2 n-2 k-1} y_{\tau}(s) f\left[s, y_{\tau}(s)\right] d s=0 .
$$

Since $y_{2 k+1}(s)>0$, there is a constant $C>0$ for which $y_{\tau}(s) \geqq C s^{2 k}$. Moreover, if $k \geqq n / 2$,

$$
\int_{T_{1}}^{1} s^{2 n-2 k-1} D^{n}\left[r(s) D^{n} y(s)\right] d s=\left[P_{1}(s)\right]_{T_{1}}^{t_{1}}-\left[(2 n-2 k-1) ! y_{2 k}(s)\right]_{T_{1}}^{t_{1}}
$$

where

$$
\begin{aligned}
P_{1}(s)= & s^{2 n-2 k-1} y_{2 n-1}(s) \\
& +\sum_{j=2}^{2 n-2 k-1}(-1)^{j+1}(2 n-2 k-1) \cdots(2 n-2 k+1-j) s^{2 n-2 k-j} y_{2 n-j}(s) .
\end{aligned}
$$

If $k<n / 2$, we have

$$
\int_{T_{1}}^{1} s^{2 n-2 k-1} D^{n}\left[r(s) D^{n} y(s)\right] d s \geqq\left[P_{2}(s)\right]_{T_{1}}^{t_{1}}-\left[M(2 n-2 k-1) ! y_{2 k}(s)\right]_{T_{1}}^{t_{1}},
$$


where

$$
\begin{aligned}
P_{2}(s)= & s^{2 n-2 k-1} y_{2 n-1}(s) \\
& +\sum_{j=2}^{n}(-1)^{j+1}(2 n-2 k-1) \cdots(2 n-2 k+1-j) s^{2 n-2 k-j} y_{2 n-j}(s) \\
& -M \sum_{j=n+1}^{2 n-2 k-1}(-1)^{j}(2 n-2 k-1) \cdots(2 n-2 k+1-j) s^{2 n-2 k-j} y_{2 n-j}(s) .
\end{aligned}
$$

We consider the products $(-1)^{j+1} y_{2 n-j}(t), j=2, \cdots, 2 n-2 k-1$. Letting $l=2 n-j,(-1)^{j+1}=(-1)^{2 n-l+1}=(-1)^{-l+1}=(-1)^{l+1}$.

Thus

$$
(-1)^{j+1} y_{2 n-j}(t)=(-1)^{l+1} y_{l}(t)>0, l=2 k+1, \cdots, 2 n-2
$$

since $y(t)$ is a $B_{k}$-solution of $H_{+}$. So each term of the sum(s) in (2.3) or (2.4) is positive. Substituting the estimates above in (2.2), we have

$$
C \int_{T_{1}}^{t} s^{2 n-1} f\left(s, C s^{2 k}\right) d s \leqq\left\{\begin{array}{c}
P_{1}\left(T_{1}\right)+(2 n-2 k-1) ! y_{2 k}(t) \\
P_{2}\left(T_{1}\right)+M(2 n-2 k-1) ! y_{2 k}(t)
\end{array}\right.
$$

which is in contradiction to (2.1) for large $t$ if $y_{2 k}(t)$ is bounded.

Letting $k=0$, we obtain a familiar criterion for the nonexistence of bounded positive solutions of $\mathrm{H}_{+}$, and hence for bounded negative solution of $H_{+}$by condition (iii). We state this as a corollary.

Corollary 2.5. Let $\tau(t)$ satisfy (T2) or (T3). Suppose that for all $C>0$

$$
\int^{\infty} t^{2 n-1} f(t, C) d t=+\infty
$$

Then all bounded solutions of $H_{+}$are oscillatory.

This corresponds to Theorem 4.1 of Ladas (1971a) in the case of the simpler equation

$$
D^{n} y(t)+p(t) f[y(t), y(g(t))]=0,
$$

where $p(t)$ is a positive continuous function on $[0, \infty), f \in C[R \times R, R], g(t) \in$ $C[[0, \infty), R], g(t) \leqq t$ for $t \geqq 0$ and $\lim _{t \rightarrow \infty} g(t)=+\infty$.

Criteria for the exclusion of $B_{i}$-solutions of $H_{-}$are formulated in this section. 
THEOREM 3.1. Let $\tau(t)$ satisfy (T2) or (T3). Suppose that for some $k=1, \cdots, n-1$ and for all $C>0$

$$
\int^{\infty} t^{2 n-2} f\left(t, C t^{2 k-1}\right) d t=+\infty .
$$

Then $H_{-}$has no solutions of type $\mathscr{B}_{k}$. If (2.1) holds with $k=0$ and $y(t)$ is a solution of $H_{-}$of type $\mathscr{B}_{0}$, then $y(t)$ tends to zero as $t \rightarrow \infty$.

Proof. Let $y(t)$ be a positive solution of $H_{-}$of type $\mathscr{B}_{k}$. Then there is a $T_{0}>0$ such that $y_{j}(t)>0$ for $j=0, \cdots, 2 k$ and $(-1)^{j} y_{j}(t)>0$ for $j=$ $2 k+1, \cdots, 2 n-1$ provided $t \geqq T_{0}$. Let $t \geqq T_{1}$ and $i \leqq n$. Integrating $H_{-} i$ times over $(t, b)$ results in

$$
y_{2 n-i}(t)=\sum_{j=0}^{i-1} \frac{y_{2 n-i+j}(b)}{j !}(t-b)^{j}
$$

$$
+\frac{1}{(i-1) !} \int_{b}^{1}(t-s)^{i-1} y_{\tau}(s) f\left[s, y_{r}(s)\right] d s
$$

i.e.,

$$
\begin{aligned}
y_{2 n-i}(t)= & \sum_{j=0}^{i-1} \frac{(-1)^{j} y_{2 n-i+j}(b)}{j !}(b-t)^{i} \\
& +\frac{(-1)^{i}}{(i-1) !} \int_{t}^{b}(s-t)^{i-1} y_{\tau}(s) f\left[s, y_{\tau}(s)\right] d s .
\end{aligned}
$$

Since (3.2) is valid for $i=n$ and $y_{n}(t) \leqq M_{0} D y_{n-1}(t)$, where $M_{0}=M$ if $y_{n}(t)>0$ and $M_{0}=m$ if $y_{n}(t)<0$, it follows that

$$
\begin{aligned}
D y_{n-1}(t) \geqq & \frac{1}{M_{0}} \sum_{j=0}^{n-1} y_{n+j}(b) \frac{(t-b)^{j}}{j !} \\
& +\frac{1}{M_{0}(n-1) !} \int_{b}^{t}(t-s)^{n-1} y_{\tau}(s) f\left[s, y_{\tau}(s)\right] d s .
\end{aligned}
$$

In the case $i>n$, an additional $i-n$ integrations of this will result in the analogous inequality

$$
\begin{aligned}
y_{2 n-i}(t) \geqq & \sum_{j=0}^{i-n-1} \frac{(-1)^{j} y_{2 n-i+j}(b)}{j !}(b-t)^{j} \\
& +\frac{1}{M_{0}} \sum_{j=i-n_{-}}^{i-1} \frac{(-1)^{j} y_{2 n-i+j}(b)}{j !}(b-t)^{j} \\
& +\frac{(-1)^{i}}{M_{0}(i-1) !} \int_{t}^{b}(s-t)^{i-1} y_{\tau}(s) f\left[s, y_{\tau}(s)\right] d s .
\end{aligned}
$$


If $k \geqq n / 2,2(n-k) \leqq n$ and we may let $i=2(n-k)$ in (3.3). Similarly, if $k<n / 2$, we may let $i=2(n-k)$ in (3.4). In either case, $2 n-i+j=2 k+j$. Each term of each sum is positive since $(-1)^{j} y_{2 k+j}(b)>0$ for $j=0, \cdots, 2 n-2 k-1$. Moreover, $y_{2 k+1}(t)<0$ for $t \geqq T_{1}$, so that $y_{2 k}\left(T_{1}\right) \geqq y_{2 k}(t)$. It follows that

$$
y_{2 k}\left(T_{1}\right) \geqq \omega_{k}^{-1} \int_{t}^{b}(s-t)^{2 n-2 k-1} y_{\tau}(s) f\left[s, y_{\tau}(s)\right] d s,
$$

where

$$
\omega_{k}=\left\{\begin{array}{cc}
(2 n-2 k-1) ! & \text { if } k \geqq n / 2 \\
M_{0}(2 n-2 k-1) ! & \text { if } k<n / 2 .
\end{array}\right.
$$

If $k=1, \cdots, n-1$, then $\lim _{t \rightarrow \infty} y_{2 k-1}(t)=\infty$; so there is a $C>0$ such that $y(t)>C t^{2 k-1}$ for $t \geqq T_{1}$. By Lemma $1.2^{\prime}$ there is a $k_{0}>0$ and a $T_{2} \geqq T_{1}$ such that

$$
y_{\tau}(t) \geqq k_{0} y(t) \geqq k_{0} C t^{2 k-1}, t \geqq T_{2} .
$$

Thus, for $k=1, \cdots, n-1$ and $s \geqq T_{2}$

$$
(s-t)^{2 n-2 k-1} y_{\tau}(s) \geqq(s-t)^{2 n-2 k-1} k_{0} C s^{2 k-1} .
$$

By (ii) $f\left[s, y_{\tau}(s)\right] \geqq f\left(s, k_{0} C s^{2 k-1}\right)$ for $s \geqq T_{2}$. Furthermore, $s-t \geqq s / 2$ for $s \geqq 2 t$. Now let $t \geqq T_{2}$ and $b>2 t=T_{*}$. It follows that

$$
\begin{aligned}
y_{2 k}\left(T_{1}\right) & \geqq k_{0} C \omega_{k}^{-1} \int_{1}^{b}(s-t)^{2 n-2 k-1} s^{2 k-1} f\left(s, k_{0} C s^{2 k-1}\right) d s \\
& \leqq k_{0} C \omega_{k}^{-1} 2^{-2 n+2 k+1} \int_{T_{0}}^{b} s^{2 n-2} f\left(s, k_{0} C s^{2 k-1}\right) d s
\end{aligned}
$$

and

$$
\int_{T_{*}}^{b} s^{2 n-2} f\left(s, k_{0} C s^{2 k-1}\right) d s \leqq 2^{2 n-2 k-1} \omega_{k}\left(k_{0} C\right)^{-1} y_{2 k}\left(T_{1}\right),
$$

which is incompatible with (3.1).

If $k=0$ and $\lim _{t \rightarrow \infty} y(t)=C>0$, then $y_{\tau}(t) \geqq y(t) \geqq C$ for $t \geqq T_{1}$ since $y^{\prime}(t)<0$ if $y(t)$ is of type $\mathscr{B}_{0}$. By (ii) $f\left[t, y_{\tau}(t)\right] \geqq f(t, C)$ for $t \geqq T_{1}$. For $t \geqq T_{1}$ and $b>2 t=T_{*}$ it follows as before that

$$
\int_{T_{*}}^{b} s^{2 n-1} f(s, C) d s \leqq 2^{2 n-1} \omega_{k} C^{-1} y_{2 k}\left(T_{1}\right),
$$

which is again a contradiction.

In the next corollary, as in the preceding theorem, we assume tacitly that $n \geqq 2$. 
COROllary 3.2. Let $\tau(t)$ satisfy (T2) or (T3). Suppose that for some $k=1, \cdots, n-1$ and for all $C>0$

$$
\int^{\infty} t^{2 n-1} f\left(t, C t^{2 k}\right) d t=+\infty .
$$

Then $H_{-}$has no $\mathscr{B}_{k}$-solutions $y(t)$ such that $\lim _{t \rightarrow \infty} y_{2 k}(t)=\gamma>0$.

THEOREM 3.3. Let $\tau(t)$ satisfy (T2) or (T3). Suppose that for all $B>0$ and $m=2 n-i, i=1,2,3$

$$
\lim _{b \rightarrow \infty} t^{-m} \int_{1}^{b} s^{2 n-1+m} f\left(s, B s^{m}\right) d s=\infty .
$$

Then $H_{-}$has no solutions of type $\mathscr{B}_{n-1}$ or $\mathscr{B}_{n}$ which are asymptotic to $C t^{m}$, where $C>0$.

Proof. Let $y(t)$ be a $\mathscr{B}_{n}$-solution of $H_{-}$. Then for $T_{1} \leqq t \leqq s \leqq b$

$$
\begin{aligned}
y(t) \geqq & \sum_{k=0}^{n-1} \frac{(-1)^{k} y_{k}(b)}{k !}(b-t)^{k}+\frac{1}{M_{0}} \sum_{k=n}^{2 n-1} \frac{(-1)^{k} y_{k}(b)}{k !}(b-t)^{k} \\
& +\frac{1}{M_{0}(2 n-1) !} \int_{t}^{b}(s-t)^{2 n-1} y_{\tau}(s) f\left[s, y_{\tau}(s)\right] d s,
\end{aligned}
$$

which is (3.4) with $i=2 n$. Since $(-1)^{k} y_{k}(b) \geqq 0$ for $k$ even,

$$
\begin{aligned}
t^{-m} y(t) \geqq & t^{-m} \sum_{j=0}^{p} \frac{y_{2 j+1}(b)}{(2 j+1) !}(t-b)^{2 j+1} \\
& +\frac{1}{M_{0}} t^{-m} \sum_{j=p}^{n-4} \frac{y_{2 j+1}(b)}{(2 j+1) !}(t-b)^{2 j+1} \\
& +\frac{1}{M_{0}(2 n-1) ! t^{m}} \int_{t}^{b}(s-t)^{2 n-1} y_{\tau}(s) f\left[s, y_{\tau}(s)\right] d s
\end{aligned}
$$

where $p=[(n-2) / 2]$. Suppose that $y(t) \sim C t^{m}, C>0$. Then there is a $T_{2} \geqq T_{1}$ such that

$$
1 / 2 \leqq y(t) / C t^{m} \leqq 3 / 2, t \geqq T_{2} .
$$

Using a familiar Tauberian theorem (See Lemma 2.2 of Wong (1969), for example.), there is a $\lambda>0$ and a $T^{*}>0$ such that

$$
\left|t^{-m} \sum_{j=0}^{p} \frac{y_{2 j+1}(b)}{(2 j+1) !}(t-b)^{2 j+1}+\frac{1}{M_{0}} t^{-m} \sum_{j=p}^{n-1} \frac{y_{2 j+1}(b)}{(2 j+1) !}(t-b)^{2 j+1}\right| \leqq \lambda
$$

for $b \geqq T^{*}$. We see that 


$$
\frac{3}{2} C t^{m} \geqq y(t) \geqq y_{\tau}(t) \geqq \frac{C}{2}[t-\tau(t)]^{m}, t \geqq T_{3},
$$

where $T_{3}=\min \left\{t>0: t-\tau(t) \geqq T_{2}, t \geqq T_{3}\right\}$. If $\tau(t)$ satisfies (T3), then $t-\tau(t) \geqq$ $(1-\mu) t$, on the other hand, if $\tau(t)$ satisfies (T2), there is a $T_{4} \geqq T_{3}$ such that $t-\tau(t) \geqq t / 2$ for $t \geqq T_{4}$ and

$$
y_{\tau}(t) \geqq \nu \frac{C}{2} t^{m}, t \geqq T_{4},
$$

where

$$
\nu=\left\{\begin{aligned}
(1-\mu)^{m} & \text { if } \tau(t) \text { satisfies (T3) } \\
2^{-m} & \text { if } \tau(t) \text { satisfies (T2) }
\end{aligned}\right.
$$

Let $t \geqq T_{4}, T_{*}=2 t$ and $b>\max \left(T_{*}, T^{*}\right)$. Then

$$
\begin{gathered}
\frac{3}{2} C \geqq t^{-m} y(t) \geqq-\lambda \times \omega_{0}^{-1} t^{-m} \int_{t}^{b}(s-t)^{2 n-1} y_{\tau}(s) f\left[s, y_{\tau}(s)\right] d s \\
\omega_{0}\left(\frac{3}{2} C+\lambda\right) \geqq \nu \frac{C}{2} t^{-m} \int_{t}^{b}(s-t)^{2 n-1} f\left(s, \nu C s^{m} / 2\right) d s
\end{gathered}
$$

and

$$
t^{-m} \int_{T_{*}}^{b} s^{2 n-1+m} f\left(s, C_{1} s^{m}\right) d s \leqq 2^{2 n-1} \omega_{0} C_{1}^{-1}\left(\frac{3}{2} C+\lambda\right),
$$

where $C_{1}=\nu C / 2$. Letting $b \rightarrow \infty$ we obtain a contradiction to (3.5).

When $\tau(t) \equiv 0, n=2$ and $k=0$, Theorem 3.1 reduces to the necessity of Theorem 2.1 of Wong (1969). When $\tau(t) \equiv 0$ and $n=2$, Theorem 3.3 reduces to the necessity of Theorem 2.3 of Wong (1969).

In this section we consider the nonhomogeneous delay equation $N_{+}$under the assumption that $R(t)$ is a solution of the ordinary differential equation

$$
D^{n}\left[r(t) D^{n} R(t)\right]=Q(t) .
$$

This permits the transformation of $N_{+}$to a homogeneous delay differential equation of order $2 n$ for which the methods of the previous selections may be applied. Since the resulting delay equation does not have exactly the same form as $H_{+}$or $H_{-}$the arguments have to be duplicated but are entirely analogous. Let us assume that $y(t)$ is a positive solution of $N_{+}$and let $u(t)=y(t)-R(t)$. Then 


$$
\begin{aligned}
D^{n}\left[r(t) D^{n} u(t)\right] & =D^{n}\left[r(t) D^{n} y(t)\right]-D^{n}\left[r(t) D^{n} R(t)\right] \\
& =-y_{\tau}(t) f\left[t, y_{\tau}(t)\right] \\
& =-(u+R)_{\tau}(t) f\left[t,(u+R)_{\tau}(t)\right],
\end{aligned}
$$

so that $u(t)$ is a solution of the homogeneous equation

$$
D^{n}\left[r(t) D^{n} u(t)\right]+(u+R)_{\tau}(t) f\left[t,(u+R)_{\tau}(t)\right]=0 .
$$

Since $y(t)>0, t \geqq T_{0},(u+R)_{\tau}(t)>0$ for $t \geqq T_{1}$ and $D^{n}\left[r(t) D^{n} u(t)\right]<0$ for $t \geqq T_{1}$ so that $u(t)$ is a nonoscillatory solution of $H_{+}^{+}$. Either $u(t)>0$ (i.e., $y(t)>R(t))$ or $u(t)<0(y(t)<R(t))$ for sufficiently large $t$. If $u(t)$ is a positive solution of $H_{+}^{+}$of type $B_{i}(j=0, \cdots, n-1)$, we will say that $y(t)$ is a solution of $N_{+}$of type $B_{j}^{R}(j=0, \cdots, n-1)$. If $u(t)<0$, then we further transform the equation by letting $v(t)=-u(t)$. It follows that $v(t)$ is a positive solution of $\left(H_{+}^{-}\right)$

$$
D^{n}\left[r(t) D^{n} v(t)\right]-(R-v)_{\tau}(t) f\left[t,(R-v)_{\tau}(t)\right]=0 .
$$

If $v(t)$ is of type $\mathscr{B}_{i}(j=0, \cdots, n)$, we will say that $y(t)$ is a solution of $N_{+}$of type $\mathscr{B}_{i}{ }_{i}$. A solution of $N_{+}$is then either oscillatory, negative nonoscillatory, of type $B_{j}^{R}(j=0, \cdots, n-1)$ or of type $\mathscr{B}_{j}^{R}(j=0, \cdots, n)$. We now seek to exclude solutions of $N_{+}$of types $B_{j}^{R}$ and $\mathscr{B}_{j}^{R}$.

THEOREM 4.1. Let $\tau(t)$ satisfy (T2) or (T3). Let $R(t)$ be a bounded solution of (4.1) and suppose that for some $k=1, \cdots, n-1$ and for all $C>0$

$$
\int^{\infty} t^{2 k} f\left(t, R_{r}(t)+C t^{2 k}\right) d t=\infty .
$$

Then $N_{+}$has no $B_{j}^{R}$ solutions for $j=k, \cdots, n-1$.

Proof. Let $y(t)$ be a positive solution of $N_{+}$of type $B_{k}^{R}$. Then $u(t)=$ $y(t)-R(t)$ is a $B_{k}$-solution of equation $H_{+}^{+}$. Let $z(t)=u_{2 n-1}(t) / u_{2 k}(t) ; z(t)$ is positive for all $t$ sufficiently large, i.e., $t \leqq T_{1}$. It follows upon differentiating $z(t)$ that

$$
z^{\prime}(t)+\frac{u_{2 n-1}(t) D u_{2 k}(t)}{\left[D u_{2 k}(t)\right]^{2}}-\frac{D u_{2 n-1}(t)}{u_{2 k}(t)}=0 .
$$

Since $u(t)$ is of type $B_{k}, u_{2 n-1}(t)$ and $D u_{2 k}(t)$ are both positive. Moreover, $D u_{2 n-1}(t)=D^{n}\left[r(t) D^{n} u(t)\right]$. Thus

$$
z^{\prime}(t)+\frac{(u+R)_{\tau}(t)}{u_{2 k}(t)} f\left[t,(u+R)_{\tau}(t)\right] \leqq 0, t \geqq T_{1} .
$$

We now consider the term 


$$
\frac{(u+R)_{\tau}(t)}{u_{2 k}(t)}=\frac{(u+R)_{\tau}(t)}{u_{\tau}(t)} \frac{u_{\tau}(t)}{u_{2 k}(t)} .
$$

By Lemma $2.1, t^{2 k} u_{2 k}(t) \leqq N u(t)$, where

$$
N=\prod_{i=0}^{2 k-1} N_{2 k-i, 2 k-i-1} .
$$

Then, assuming $\tau(t)$ satisfies (T3)

$$
(1-\mu)^{2 k} t^{2 k} u_{2 k . \tau}(t) \leqq[t-\tau(t)]^{2 k} u_{2 k, \tau}(t) \leqq N u_{\tau}(t)
$$

If $\tau(t)$ satisfies (T2), then $\mu t^{\beta-1}<1 / 2$ for $t$ sufficiently large, so that

$$
t-\tau(t) \geqq t-\mu t^{\beta}=t\left(1-\mu t^{\beta-1}\right) \geqq \frac{t}{2}
$$

for $t \geqq T_{2} \geqq T_{1}$. It follows that

$$
u_{\tau}(t) \geqq \mu_{k} N^{-1} t^{2 k} u_{2 k_{\tau} \tau}(t),
$$

where

$$
\mu_{k}=\left\{\begin{array}{ll}
2^{-2 k} & \text { if } \tau(t) \text { satisfies (T2) } \\
(1-\mu)^{2 k} & \text { if } \tau(t) \text { satisfies (T3) }
\end{array} .\right.
$$

By Lemma 2.2 , there is a $k_{0}>0$ such that $u_{2 k, \tau}(t) u_{2 k}^{-1}(t) \geqq k_{0}$.

So for $t \geqq T_{3} \geqq T_{2}$

$$
\frac{u_{\tau}(t)}{u_{2 k}(t)} \geqq \mu_{k} N^{-1} k_{0} t^{2 k}
$$

Next we consider the expression

$$
\frac{(u+R)_{\tau}(t)}{u_{\tau}(t)}=\left[\frac{u+R}{u}\right]_{\tau}(t)=\left(1+\frac{R}{u}\right)_{\tau}(t) .
$$

If $R(t)$ is bounded and $y(t)$ is of type $B_{k}^{R},(k=1, \cdots, n-1)$, then $u(t)$ is unbounded, which implies that $R u^{-1} \rightarrow 0$ as $t \rightarrow \infty$. So for $\varepsilon>0,1+R u^{-1} \geqq$ $1-\varepsilon=C_{0}$ for $t$ sufficiently large. Thus there is a $T_{*} \geqq T_{3}$ such that

$$
(u+R)_{\tau}(t) u_{\tau}^{-1}(t) \geqq C_{0}, t \geqq T_{*} .
$$

Moreover, $u_{2 k}(t)$ and $u_{2 k+1}(t)$ are positive; so there is a $C_{1}>0$ such that $(u+R)_{\tau}(t) \geqq R_{\tau}(t)+C_{1} t^{2 k}$. Substitution of this estimate together with (4.4) and (4.5) via hypothesis (ii) in (4.3) yields

$$
z^{\prime}(t)+C_{0} \mu_{k} k_{0} N^{-1} t^{2 k} f\left(t, R_{\tau}(t)+C_{1} t^{2 k}\right) \leqq 0 .
$$

An integration of this from $T_{*}$ to $t$ results in 


$$
\int_{\tau_{*}}^{t} t^{2 k} f\left(t, R_{r}(t)+C_{1} t^{2 k}\right) d t \leqq\left(C_{0} \mu_{k} k_{0}\right)^{-1} N\left[z\left(T_{*}\right)-z(t)\right] \leqq\left(C_{0} \mu_{k} k_{0}\right)^{-1} N z\left(T_{*}\right),
$$

which is in contradiction to (4.2).

By hypothesis (ii), the divergence of $t^{2 k} f\left(t, R_{\tau}(t)+C_{1} t^{2 k}\right)$ implies that of $t^{2(k+i)} f\left(t, R_{r}(t)+C_{1} t^{2(k+i)}\right)$ for $i=0, \cdots, n-k-1$. So condition (4.2) is sufficient to exclude $B_{j}^{R}$-solutions $(j=k, \cdots, n-1)$ of $N_{+}$. If $n=1, j=0$ is the only possibility and the theorem is vacuously true.

By considering (4.5) more carefully we are able to obtain the following corollaries to the proof of Theorem 4.1.

COROLlaRY 4.2. Let $\tau(t)$ satisfy (T2) or (T3) and $R(t)$ be a solution of (4.1). Then $N_{+}$has no $B_{k}^{R}$-solutions $y(t)$ such that $(y-R)_{2 k}(t)$ is bounded if for all positive constants $C$

$$
\int^{\infty}\left(R_{\tau}(t)+C t^{2 k}\right) f\left(t, R_{\tau}(t)+C t^{2 k}\right) d t=\infty .
$$

Corollary 4.3. Let $\tau(t)$ satisfy (T2) or (T3) and $R(t)$ be a bounded solution of (4.1). Then $N_{+}$has no bounded $B_{k}^{R}$-solutions if for all positive constants $C$

$$
\int^{\infty}\left(R_{\tau}(t)+C\right) f\left(t, R_{\tau}(t)+C\right) d t=\infty .
$$

CoROllary 4.4. Let $\tau(t)$ satisfy (T2) or (T3) and $R(t)$ be a bounded solution of (4.1). Suppose that for all positive constants $C$

$$
\int^{\infty} f\left(t, R_{\tau}(t)+C\right) d t=\infty .
$$

Then no bounded $B_{0}^{R}$-solution of $N_{+}$is bounded way from zero as $t \rightarrow \infty$.

REMARK 1. In Theorem 4.1 the hypothesis that $R(t)$ is bounded may be replaced by $R(t)=0\left(t^{2 k-\varepsilon}\right)$, for some $\varepsilon>0$.

REMARK 2. The conclusion of Theorem 4.1 may be restated as: A positive solution $y(t)$ of $N_{+}$either satisfies $0<y(t)<R(t)$ for large $t$ or is of type $B_{i}^{R}$, where $j=0, \cdots, k-1$.

Remark 3. If $R(t)$ is oscillatory or negative, then $u(t)>0$ and the conclusion of Theorem 4.1 becomes: A positive solution of $N_{+}$is of type $B_{j}^{R}$ $(j=0, \cdots, k-1)$.

It may be easily seen that the integral condition (4.7') is sufficient to guarantee the nonexistence of all positive solutions of $N_{+}$of types $B_{k}^{R}(k=$ $0, \cdots, n-1)$. We suppose that $y(t)$ is of type $B_{k}^{R}$ on $\left(T_{*}, \infty\right)$. An integration of $H_{+}^{+}$over $\left(T_{*}, t\right)$ results in 


$$
u_{2 n-1}(t)-u_{2 n-1}\left(T_{*}\right)+\int_{T_{*}}^{t}(u+R)_{\tau}(s) f\left[s,(u+R)_{\tau}(s)\right] d s=0
$$

i.e.,

$$
\int_{T_{* 1}}^{t}(u+R)_{\tau}(s) f\left[s,(u+R)_{\tau}(s)\right] d s \leqq u_{2 n-1}\left(T_{*}\right) .
$$

Since $u^{\prime}(t)$ is positive for $t \geqq T_{*}$, there is a constant $C>0$ such that $u_{\tau}(t) \geqq C$ for $t \geqq T_{*}$ and

$$
\int_{T_{*}}^{l}\left(R_{\tau}(s)+C\right) f\left(s, R_{\tau}(s)+C\right) d s \leqq u_{2 n-1}\left(T_{*}\right) .
$$

The divergence of the integral above as $t \rightarrow \infty$ will result in a contradiction. We have thus proved the following theorem:

Theorem 4.5. Let $\tau(t)$ satisfy (T2) or (T3). Let $R(t)$ be a solution of (4.1) and suppose that for all $C>0$

$$
\int^{\infty}\left(R_{\tau}(t)+C\right) f\left(t, R_{\tau}(t)+C\right) d t=\infty .
$$

Then $N_{+}$has no $B_{j}^{R}$-solutions $(j=0, \cdots, n-1)$.

REMARK 4. If $R(t)$ is oscillatory or negative, the conclusion of Theorem 4.5 may be strengthened to: $N_{+}$has no positive solutions. If, in addition, it is assumed that for all $C>0$

$$
\int^{\infty}\left(R_{\tau}(t)-C\right) f\left(t, R_{\tau}(t)-C\right) d t=-\infty
$$

then $N_{+}$cannot have any negative solutions for large $t$, i.e., $N_{+}$is oscillatory. The result is essentially part of a theorem due to Kartsatos and Manougian (to appear) provided $f(t, u)=p(t) F(u)$.

Another approach to the question of bounded solutions of $N_{+}$results from applying to equation $\mathrm{H}_{+}^{+}$the method used to obtain Theorem 2.4 and Corollary 2.5. We obtain the analogous results:

THEOREM 4.6. Let $\tau(t)$ satisfy (T2) or (T3) and $R(t)$ be a solution of (4.1). Suppose that for some $k=0, \cdots, n-1$ and for all positive constants $C$

$$
\int^{\infty} t^{2 n-2 k-1}\left(R_{\tau}(t)+C\right) f\left(t, R_{\tau}(t)+C\right) d t=\infty .
$$

Then $N_{+}$has no $B_{k}^{R}$-solutions $y(t)$ for which $[y(t)-R(t)]_{2 k}$ is bounded.

COROLlary 4.7. Let $\tau(t)$ satisfy (T2) or (T3) and $R(t)$ be a bounded solution of (4.1). Suppose that (4.8) holds with $k=0$. Then $N_{+}$has no bounded positive $B_{k}^{R}$-solutions. 
The corollary follows upon observing that since $R(t)$ is bounded, a $B_{k}^{R}$-solution $y(t)$ is bounded if, and only if, $u(t)$ is bounded. For $k \geqq 1, u(t)$ is unbounded by Lemma 2.1. The case $k=0$ is excluded by Theorem 4.6.

ReMARK 5. In view of Remarks 3 and 4 we may assume, without loss of generality, that $R(t)>0$ in attempting to exclude $\mathscr{B}_{j}^{R}$-solutions of $N_{+}$.

THEOREM 4.8. Let $\tau(t)$ satisfy (T2) or (T3) and $R(t)$ be a positive solution of (4.1). Suppose that for some $k=0, \cdots, n-1$ and for all $C>0$

$$
\int^{\infty} t^{2 n-2 k-1} f(t, C) d t=\infty .
$$

Then no positive solution of $N_{+}$of type $\mathscr{B}_{k}^{R}$ is bounded away from zero as $t \rightarrow \infty$.

Proof. Let $y(t)$ be a positive solution of $N_{+}$of type $\mathscr{B}_{k}^{R}$ on $\left[T_{0}, \infty\right)$. Then $v(t)=R(t)-y(t)$ is a positive solution of $H_{+}^{-}$of type $\mathscr{B}_{k}$ on $\left[T_{0}, \infty\right)$ and $D v_{2 n-1}(t)$ for $t \geqq T_{1}$. Suppose that $y(t)$ is bounded away from zero as $t \rightarrow \infty$. Let $t \geqq T_{1}, b>2 t$ and $i \leqq n$. Integration of $H_{+}^{-}$over $(t, b)$ results in

$$
v_{2 n-i}(t)=\sum_{j=0}^{i-1}(-1)^{j} v_{2 n-i+j}(b) \frac{(b-t)^{j}}{j !}
$$

$$
+\frac{(-1)^{i}}{(i-1) !} \int_{t}^{b}(s-t)^{i-1}(R-v)_{\tau}(s) f\left[s,(R-v)_{\tau}(s)\right] d s .
$$

For the case $i>n$, we obtain

$$
\begin{aligned}
v_{2 n-i}(t) \geqq & \sum_{j=0}^{i-n-1}(-1)^{j} v_{2 n-i+j}(b) \frac{(b-t)^{j}}{j !} \\
& +\frac{1}{M_{0}} \sum_{,-i-n}^{i-1}(-1)^{j} v_{2 n-i+j}(b) \frac{(b-t)^{j}}{j !} \\
& +\frac{(-1)^{i}}{M_{0}(i-1) !} \int_{t}^{b}(s-t)^{i-1}(R-v)_{\tau}(s) f\left[s,(R-v)_{\tau}(s) d s .\right.
\end{aligned}
$$

Letting $i=2(n-k)$ in $(4.10)$ if $k \geqq n / 2$ or in $\left(4.10^{\prime}\right)$ if $k<n / 2$, we obtain

$$
\begin{aligned}
v_{2 k}\left(T_{1}\right) & \geqq v_{2 k}(t) \geqq \omega_{k}^{-1} \int_{T}^{b}(s-t)^{2 n-2 k-1}(R-v)_{\tau}(s) f\left[s,(R-v)_{\tau}(s)\right] d s \\
& \geqq 2^{2 k+1-2 n} \omega_{k}^{-1} \int_{T}^{b} s^{2 n-2 k-1}(R-v)_{\tau}(s) f\left[s,(R-v)_{\tau}(s)\right] d s .
\end{aligned}
$$


Since $y(t)=R(t)-v(t)$ is bounded away from zero as $t \rightarrow \infty$, there is a constant $C>0$ such that $(R-v)_{\tau}(t) \geqq C$ for $t \geqq T$. Thus

$$
\int_{T}^{b} s^{2 n-2 k-1} f(s, C) d s \leqq 2^{2 n-2 k-1} \omega_{k} C^{-1},
$$

which is in contradiction to (4.9) as $b \rightarrow \infty$.

We now apply some of the theorems above to investigate the asymptotic behavior of bounded solutions of the ordinary differential equation

$$
\left(D^{4}+1\right) y(t)=e^{-t} \text {. }
$$

The general solution of (4.11) is given by

$$
\begin{aligned}
y_{N}(t)= & c_{1} e^{t / \sqrt{2}} \cos \left(t / \sqrt{2}+c_{2} e^{t / \sqrt{2}} \sin (t / \sqrt{2})+c_{3} e^{-t / \sqrt{2}} \cos (t / \sqrt{2})\right. \\
& +c_{4} e^{-t / \sqrt{2}} \sin (t / \sqrt{2})+\frac{1}{2} e^{-t} .
\end{aligned}
$$

Since $e^{-t / \sqrt{ } 2}>e^{-t}$, a bounded solution $y(t)$ of (4.11) is either oscillatory (if $c_{1}^{2}+c_{2}^{2}+c_{3}^{2}+c_{4}^{2} \neq 0$ ) or else positive nonoscillatory. In either event, $\lim _{t \rightarrow x} y(t)=0$.

We have $Q(t)=e^{-t}$ and $r(t) \equiv 1$, the general solution of $D^{4} R(t)=e^{-t}$ is

$$
R(t)=e^{-t}+a_{1} t^{3}+a_{2} t^{2}+a_{3} t+a_{4} .
$$

Let us choose $R(t)=e^{-t}$ and let $y(t)$ be a bounded solution of (4.11). If $y(t)$ is a $B_{k}^{R}$-solution, then $0<y(t)<R(t)$ and $\lim _{t \rightarrow \infty} y(t)=0$. This fact is confirmed by Theorem 4.8 in the case $k=0,1$ since

$$
\infty=\int^{\infty} t^{2 n-2 k-1} f(t, C) d t=\left\{\begin{array}{ll}
\int^{\infty} t^{3} d t & \text { if } k=0 \\
\int^{\infty} t d t & \text { if } k=1
\end{array} .\right.
$$

If $y(t)$ is of type $B_{1}^{R}$, then $y(t)-R(t)$ is necessarily unbounded; since $R(t)$ is bounded, this would imply that $y(t)$ is unbounded. Theorem 4.1 shows that there are, in fact, no $B_{1}^{R}$-solutions:

$$
\int^{\infty} t^{2}\left(e^{-(t-\tau(t))}+C\right) d t=\infty .
$$

Corollary 4.4 shows that no $B_{0}^{R}$-solution is bounded away from zero since

$$
\int^{\infty}\left[e^{-(t-\tau(t))}+C\right) d t=\infty .
$$


We now consider the nonhomogeneous delay equation $N_{-}$under the assumption that $R(t)$ is a solution of the equation (4.1). As in the previous section, this permits the transformation of $N_{-}$to a homogeneous delay equation for which the techniques of sections two and three are applicable.

Let us assume that $y(t)$ is a positive solution of $N_{-}$and let $u(t)=$ $y(t)-R(t)$. Then

$$
\begin{aligned}
D^{n}\left[r(t) D^{n} u(t)\right] & =D^{n}\left[r(t) D^{n} y(t)\right]-D^{n}\left[r(t) D^{n} R(t)\right] \\
& =y_{\tau}(t) f\left[t, y_{\tau}(t)\right]
\end{aligned}
$$

so that $u(t)$ is a solution of the homogeneous equation

$$
D^{n}\left[r(t) D^{n} u(t)\right]-(u+R)_{\tau}(t) f\left[t,(u+R)_{\tau}(t)\right]=0 .
$$

Since $y(t)>0,(u+R)_{\tau}(t)$ is positive for sufficiently large $t\left(t \geqq T_{1}\right)$ and $D^{n}\left[r(t) D^{n} u(t)\right]>0$ for $t \geqq T_{1}$. It follows that $u(t)$ is a nonoscillatory solution of $H_{-}^{-}$. Either $u(t)>0$ or $u(t)<0$. If $u(t)<0$, we further transform the equation by letting $v(t)=-u(t)=R(t)-y(t)$. Then $v(t)$ is a positive solution of

$$
D^{n}\left[r(t) D^{n} v(t)\right]+(R-v)_{\tau}(t) f\left[t,(R-v)_{\tau}(t)\right]=0 .
$$

We say that a positive solution $y(t)$ of $N_{-}$is of type $\mathscr{B}_{j}^{R}$ if $u(t)$ is a positive solution of $\boldsymbol{H}_{-}^{-}$of type $\mathscr{B}_{j}$; it is of type $B_{j}^{R}$ if $v(t)$ is a positive solution of $\boldsymbol{H}_{-}^{+}$of type $B_{j}$. A positive solution of $N_{-}$is then of type $\mathscr{B}_{j}^{R}(j=0, \cdots, n)$ or of type $B_{i}{ }_{i}$ $(j=0, \cdots, n-1)$. We now seek to exclude $\mathscr{B}_{i}{ }_{i}$ - and $B_{i}{ }_{i}$-solutions of $N_{\text {. }}$.

THEOREM 5.1. Let $\tau(t)$ satisfy (T2) or (T3) and $R(t)$ be a solution of (4.1). Suppose that for some $k=1, \cdots, n-1$ and for all $C>0$

$$
\int^{\infty} t^{2 n-2 k-1}\left(R_{\tau}(t)+C t^{2 k-1}\right) f\left(t, R_{\tau}(t)+C t^{2 k-1}\right) d t=+\infty
$$

Then $N_{-}$has no $\mathscr{B}_{k}^{R}$-solutions. Furthermore, if

$$
\int^{\infty} t^{2 n-1}\left(R_{\tau}(t)+C\right) f\left(t, R_{\tau}(t)+C\right) d t=+\infty,
$$

then any $\mathscr{B}_{0}^{R}$-solution $y(t)$ satisfies

$$
\lim _{t \rightarrow \infty}[y(t)-R(t)]=0
$$


Proof. Let $y(t)$ be a $\mathscr{B}_{k}^{R}$-solution of $N_{\text {. }}$. Then $u(t)=y(t)-R(t)$ is a positive solution of $H_{-}^{-}$of type $\mathscr{B}_{k}^{R}$. As in the proof of Theorem 4.3, we obtain

$$
\begin{aligned}
u_{2 n-i}(t)= & \sum_{i=0}^{i-1} \frac{u_{2 n-i+j}(b)}{j !}(t-b)^{j} \\
& +\frac{(-1)^{i}}{(i-1) !} \int_{t}^{b}(s-t)^{i-1}(u+R)_{\tau}(s) f\left[s,(u+R)_{\tau}(s)\right] d s,
\end{aligned}
$$

where $T_{1} \leqq t \leqq s<b$ and $i \leqq n$.

If $i>n$, we may derive the analogous inequality

$$
\begin{aligned}
u_{2 n-i}(t) \geqq & \sum_{j=0}^{i-n-1} \frac{u_{2 n-i-j}(b)}{j !}(t-b)^{j}+\frac{1}{M_{0}} \sum_{j=i-n}^{i-1} \frac{u_{2 n-i+j}(b)}{j !}(t-b)^{j} \\
& +\frac{(-1)^{i}}{M_{0}(i-1) !} \int_{t}^{b}(s-t)^{i-1}(u+R)_{\tau}(s) f\left[s,(u+R)_{\tau}(s)\right] d s .
\end{aligned}
$$

Letting $i=2(n-k)$ in the equality (5.3) if $k \geqq n / 2$ and in the inequality (5.4) if $k<n / 2$ and recalling that $D u_{2 k}(t)<0$ for $t \geqq T_{1}$, we have

$$
\int^{b}(s-t)^{2 n-2 k-1}(u+R)_{\tau}(s) f\left[s,(u+R)_{\tau}(s)\right] d s \leqq \omega_{k} u_{2 k}\left(T_{1}\right) .
$$

For $k=1, \cdots, n$ there is a constant $C>0$ such that $u_{\tau}(s) \geqq C s^{2 k-1}$ for $s \geqq 2 T_{1}=$ $T_{2}$. Let $t \geqq T_{2}$ and $b>2 t=T_{*}$. Then $s-t \geqq s / 2$ and

$$
\int_{T}^{b} s^{2 n-2 k-1}\left(R_{\tau}(s)+C s^{2 k-1}\right) f\left[s, R_{\tau}(s)+C s^{2 k-1}\right] d s \leqq 2^{2 n-2 k-1} \omega_{k} u_{2 k}\left(T_{1}\right),
$$

which is in contradiction to (5.1) as $b \rightarrow \infty$.

If $k=0$ and $y(t)-R(t)$ does not tend to zero as $t \rightarrow \infty$, there is a constant $C>0$ such that $u(t) \geqq C$ for $t$ sufficiently large $\left(t \geqq T_{*}\right)$. As before we may apply hypothesis (ii) to obtain a contradiction to $\left(5.1^{\prime}\right)$.

Duplicating the arguments which led to Theorems 3.3 and 4.4 , we may obtain the following result:

THEOREM 5.2. Let $\tau(t)$ satisfy (T2) or (T3) and let $R(t)$ be a solution of (4.1). Suppose that for some $m=2 n-i$, where $i=1,2,3$, and for all positive constants $B$

$$
\lim _{b \rightarrow \infty} t^{-m} \int_{t}^{b} s^{2 n-1}\left(R_{\tau}(s)+B s^{m}\right) f\left[s, R_{\tau}(s)+B s^{m}\right) d s=+\infty .
$$


Then $N_{-}$has no solutions of types $B_{n}^{R}$ or $B_{n-1}^{R}$ such that $R(t)-y(t) \sim C t^{m}$, where $C>0$.

If we let $w(t)=v_{2 n-1}(t) v_{2 k}^{-1}(t)$ in $H_{-}^{+}$and repeat the procedure which led to Theorems 2.3 and 4.1, we may obtain the following analogue:

THEOREM 5.3. Let $\tau(t)$ satisfy (T2) or (T3) and let $R(t)$ be a bounded solution of (4.1). Suppose that for some $k=0, \cdots, n-1$ and for all positive constants $C$

$$
\int^{\infty} t^{2 k} f(t, C) d t=\infty
$$

Then any positive $B_{k}^{R}$-solution $y(t)$ tends to zero as $t \rightarrow \infty$.

\section{Summary}

We conclude this paper with some observations on the method. Use of the preliminary transformation $Y(t)=-y(t)$ and the techniques of Sections four and five enable us to introduce a natural classification of the negative solutions of $N_{+}$and $N_{-}$and to provide sufficient conditions for the nonexistence of such solutions. If the hypothesis $f(t,-u)=f(t, u)$ is omitted, we may still determine conditions for the nonexistence of certain negative solutions. Moreover, the method is applicable even if hypothesis (ii) is replaced by (ii') there is a $\rho$ $(0<\rho<1)$ such that $u^{\rho} f(t, u)$ is nondecreasing in $u$. The precise statements and proofs of these analogous results are left to the reader to discover.

\section{References}

L. P. Burton (1952), 'Oscillation theorems for the solutions of linear, nonhomogeneous, secondorder differential systems', Pacific J. Math. 2, 281-289.

H. C. Howard (to appear), 'Oscillation and nonoscillation criteria for nonhomogeneous differential equations.'

A. G. Kartsatos (1971), 'On the maintenance of oscillations under the effect of a small forcing term', J. Differential Equations 10, 355-363.

A. G. Kartsatos (1972), 'Maintenance of oscillations under the effect of a periodic forcing term', Proc. Amer. Math. Soc. 33, 377-383.

A. G. Kartsatos and M. M. Manougian (to appear), 'Perturbations causing oscillations of functional differential equations', Proc. Amer. Math. Soc.

T. Kusano (to appear), 'Differential equations with retarded arguments', Proc. Carathéodory Symposium, Athens, Greece (1973).

T. Kusano and $H$. Onose (1974), 'Oscillations of functional differential equations with retarded argument', J. Differential Equations 15, 269-277.

G. Ladas (1971a), 'Oscillation and asymptotic behavior of solutions of differential equations with retarded arguments', J. Differential Equations 10, 281-290. 
G. Ladas (1971b), 'On principal solutions of nonlinear differential equations', J. Math. Anal. Appl. 36, $103-109$.

B. Singh (to appear), 'Asymptotically vanishing oscillatory trajectories in second order retarded equations', Siam J. Math. Anal.

B. Singh and R. S. Dahiya (to appear), 'Effect of delays on oscillation in functional differential equations with time lag', Siam J. Appl. Math.

R. D. Terry (1974), 'Oscillatory properties of a delay differential equation of even order', Pacific $J$. Math. 52, 269-282.

R. D. Terry and P. K. Wong (1972), 'Oscillatory properties of a fourth-order delay differential equation', Funkcial. Ekvac. 15, 209-221.

P. K. Wong (1969), 'On a class of nonlinear fourth order differential equations', Ann. Mat. Pura Appl. 81, 331-346.

California Polytechnic State University, San Luis Obispo,

California, U.S.A. 